\title{
A specialized motion capture system for real-time analysis of mandibular movements using infrared cameras
}

\author{
Daniel Antônio Furtado ${ }^{1 *}$, Adriano Alves Pereira', Adriano de Oliveira Andrade ${ }^{1}$, \\ Douglas Peres Bellomo Junior ${ }^{1}$ and Marlete Ribeiro da Silva ${ }^{2}$
}

\author{
* Correspondence: danielfurtados@ \\ yahoo.com.br \\ ${ }^{1}$ Laboratory of Biomedical \\ Engineering, Faculty of Electrical \\ Engineering, Federal University of \\ Uberlandia, Av. Joao Naves de Avila, \\ 2121, Uberlandia, MG CEP 38408 \\ 100, Brazil \\ Full list of author information is \\ available at the end of the article
}

\begin{abstract}
Background: In the last years, several methods and devices have been proposed to record the human mandibular movements, since they provide quantitative parameters that support the diagnosis and treatment of temporomandibular disorders. The techniques currently employed suffer from a number of drawbacks including high price, unnatural to use, lack of support for real-time analysis and mandibular movements recording as a pure rotation. In this paper, we propose a specialized optical motion capture system, which causes a minimum obstruction and can support 3D mandibular movement analysis in real-time.
\end{abstract}

Methods: We used three infrared cameras together with nine reflective markers that were placed at key points of the face. Some classical techniques are suggested to conduct the camera calibration and three-dimensional reconstruction and we propose some specialized algorithms to automatically recognize our set of markers and track them along a motion capture session.

Results: To test the system, we developed a prototype software and performed a clinical experiment in a group of 22 subjects. They were instructed to execute several movements for the functional evaluation of the mandible while the system was employed to record them. The acquired parameters and the reconstructed trajectories were used to confirm the typical function of temporomandibular joint in some subjects and to highlight its abnormal behavior in others.

Conclusions: The proposed system is an alternative to the existing optical, mechanical, electromagnetic and ultrasonic-based methods, and intends to address some drawbacks of currently available solutions. Its main goal is to assist specialists in diagnostic and treatment of temporomandibular disorders, since simple visual inspection may not be sufficient for a precise assessment of temporomandibular joint and associated muscles.

Keywords: Mandibular movements, Optical motion capture, Temporomandibular joint

\section{Background}

Temporomandibular joint (TMJ) and the human mandible are part of an interesting and complex biomechanical system capable to perform several functions and high precision movements, such as chewing, swallowing and speech [1]. Systems designed to record and analyze these movements have received increasing attention in the past

(C) 2013 Furtado et al.; licensee BioMed Central Ltd. This is an Open Access article distributed under the terms of the Creative Commons Attribution License (http://creativecommons.org/licenses/by/2.0), which permits unrestricted use, distribution, and reproduction in any medium, provided the original work is properly cited. 
few years, since they provide quantitative parameters that support the clinical diagnosis and treatment of temporomandibular disorders (TMD) [2-4].

Therefore, a number of methods, techniques and devices for recording and analyzing human mandibular movements have recently been proposed [1,5-18]. Conventional mechanical methods, such as JT-3D System from BioResearch Company, usually employ an articulated mechanism fixed on the head in order to record mandibular motion. However, most of these devices just simulate a pure rotation of lower jaw along a single axis, while mandibular movements involve simultaneous rotation and translation [19].

Ultrasonic-based methods, such as JMA System of zebris Medical GmbH, typically use a face-bow together with integrated receiver sensors for acquisition of 3D mandibular movements. In this case, motion is captured by measuring the travel time of ultrasound impulses $[14,20]$. Similarly, some electromagnetic-based techniques, like the prototype system presented by Santos et al. (2008) [1], use a facial arc with electromagnetic sensors to record appropriate kinematics. However, these magnetic methods can be sensitive to the presence of metal in the environment [21] and their accuracy has rarely been reported.

In most cases, mechanical, electromagnetic and ultrasonic-based devices are somewhat bulky and make the patients feel unnatural during the routine tests. In addition, many of them are also relatively heavy, expensive and complicated to use [16].

Another group of methods and systems used to record jaw movements works with video cameras and passive or active markers. Some authors have presented off-line techniques employing low-cost CCD cameras [5,6,9,14,19], while others have used commercial, high-end motion capture packs $[17,22,23]$. By using a single CCD camera and a reflective marker fixed to the mandible, Pinheiro et al. (2011) [6] proposed a computational method for recording mandibular movements in a two-dimensional space. With a mean error of $0.4 \mathrm{~mm}$, analyses were done separately in frontal and sagittal planes, but not in 3D. Further, Fang and Kuo (2008) [19] presented a system using a pair of CCD cameras and three light-emitting diodes (LED) affixed to a pair of tracking plates for 3D reconstruction. The main disadvantage of this system is the obstruction caused by the tracking plates, which can limit the subject's freedom when executing jaw movements. The authors reported an RMS accuracy of $0.198 \mathrm{~mm}$.

Commercial optical systems used by the entertainment industry to get facial expression, or even optical systems employed to capture full-body motion, could be used to register mandibular movements. Indeed, Rohrle et al. (2009) [17] and Mani et al. (2010) [23] have analyzed jaw movements using a Vicon MX system and a Qualisys five-camera system, respectively. The problem with this idea is the high cost of these systems, which usually include expensive and sophisticated software that is developed to work with dozens of markers and handle extreme conditions, like fast motion, marker occlusions and large capture volumes. However, none of these features is actually needed for tracking the mandible. Also, these systems are not specialized to perform mandibular motion capture and some actions, like marker identification or rigid body creation, may have to be done manually.

In this context, we propose a specialized optical motion capture system for mandibular movement analysis using three infrared cameras and a set of nine reflective markers. Unlike most techniques and devices recently published in literature, the proposed system brings together a set of important features, once it combines good precision and accuracy, minimum obstruction, real-time 3D reconstruction 
and analysis and moderate cost. Furthermore, it can give parameters of facial morphology and can automatically recognize the markers.

More specifically, our contribution includes the proposal of a reflective marker setup together with a camera set configuration, the suggestion and evaluation of some classical, already available algorithms for camera calibration and 3D reconstruction in the context of mandibular movement analyzing, and the proposal of a specialized computational method to perform automatic identification and tracking of our set of markers in real time. All these algorithms and methods are relatively simple, as they were implemented and evaluated in our prototype software. The validation test suggests a mean error of $0.156 \mathrm{~mm}$ and a precision of $0.259 \mathrm{~mm}$ within the volume intended for recording mandibular movement.

\section{Methods}

To perform three-dimensional reconstruction of condylar movements, we propose the usage of three infrared specialized cameras and a set of reflective markers that must be arranged at key points of the subject's face. In our experiments, we have used cameras model OptiTrack Flex V100, manufactured by NaturalPoint. These cameras are natively capable to find out white points in the images, which correspond to reflective objects (usually markers) in the scene. All cameras synchronously take images of the scene and reduce the image data to a set of $2 \mathrm{D}$ image coordinates representing the detections of the markers. Each camera is able to capture up to 100 frames per second, which is sufficiently high to guarantee detailed register of lower jaw movements. It is important to note that we have used only the three infrared cameras, which cost just a fraction of the whole motion capture system offered by the manufacturer (the complete system includes a 3D reconstruction software, calibration tools, USB hubs, etc.).

\section{Marker set}

A set of nine retro-reflective markers is proposed to allow mandibular movement analysis. Eight of them are called the secondary tracking markers and their purpose includes estimating some morphological parameters of the mandible. The primary tracking marker is the one primarily employed to track the movement of the jawbone.

Secondary tracking markers can be fixed on skin by using adhesive tape and a plastic support. They must be positioned on the following regions of the face: (1) TMJ external surface (left and right), (2) mandible angle region (left and right), (3) middle region between the chin and the mandible angle (left and right), (4) above upper lip and (5) on the forehead. Figure 1 illustrates these points.

Due to the movement of the skin over the bones, 3D coordinates of the secondary markers do not exactly correspond to the real positions of the underlying bones. However, these coordinates can be used to support the alignment of the head, to provide a visual reference for three-dimensional analysis and to give a rough approximation of the facial morphology of the subject, since facial morphology can influence condylar movements [3,24]. Therefore, mandibular parameters such as gonial angle, bigonial width and bicondylar width can be estimated from the reconstructed 3D coordinates of the secondary markers. 


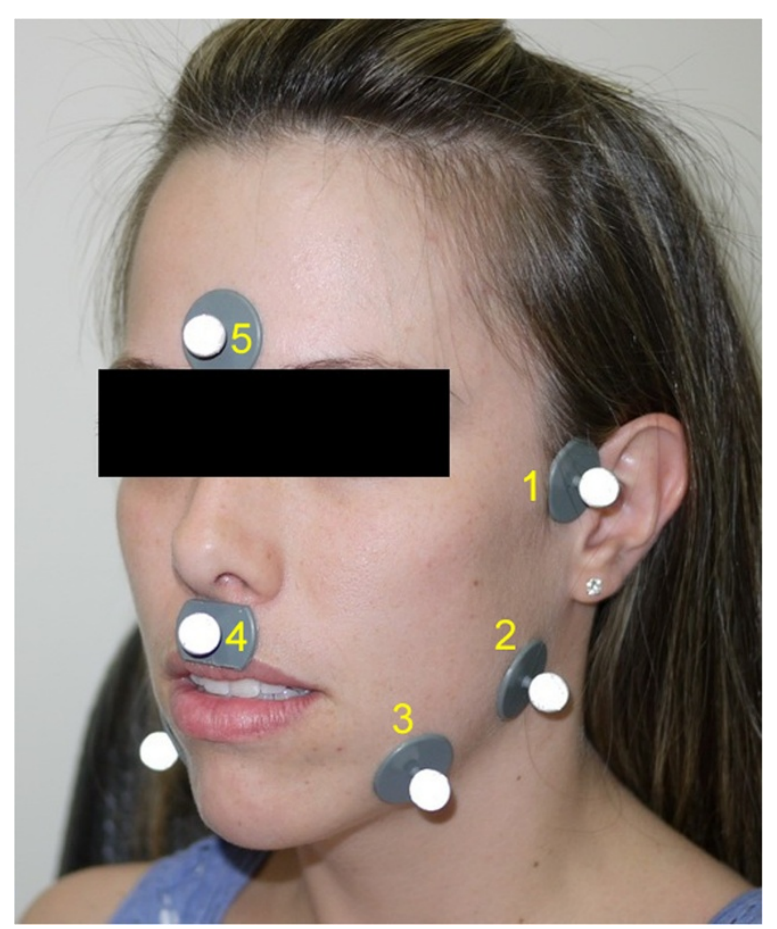

Figure 1 Proposed marker setup. (1) TMJ external surface; (2) mandible angle region; (3) middle region between the chin and the mandible angle; (4) above upper lip and (5) on the forehead. Markers in positions 1, 2 and 3 are placed on both sides of the face. Smaller ones could also be used.

To maximize accuracy and precision, the primary tracking marker must be set firmly to the mandible. In our experiments, we have employed a metal support fixed inside the mouth. The support was placed between the inferior lip and the labial surface of the lower incisors, as illustrated in Figure 2. A thermoplastic material (godiva) was used to make the fixing base and a zinc-enolic paste $\left(\right.$ Lyzanda $\left.^{\circledR}\right)$ was employed as adhesive material making the interface between teeth and the base. As a result, the path traveled by the primary marker is truly related to the path traveled by the mandibular condyle [13].

The forehead marker aims to estimate the movement of the head. In fact, mandibular movements are a combination of condylar and head movements [25]. Therefore, the movement of the incisive point can be compensated by subtracting the movement of the marker on the forehead from the movement of the primary marker [6].

\section{Camera configuration}

In order to allow $3 \mathrm{D}$ reconstruction, we need each marker to be seen by at least two cameras. Considering our set of markers, this condition can be satisfied by placing one camera in front of the subject (to see all markers) and one on each side (left and right). The proposed configuration is presented in Figure 3.

The left and right cameras must be placed at about 1 meter distance from the subject, forming an angle of approximately 120 degrees and the central camera should be located at 1.3 meters from the subject. The three cameras must be vertically positioned about $20 \mathrm{~cm}$ above the head line. With this camera configuration, the dental specialist can freely move himself around the subject while the shot distances are still sufficient to ensure good precision and accuracy when detecting the markers. Additionally, this arrangement 


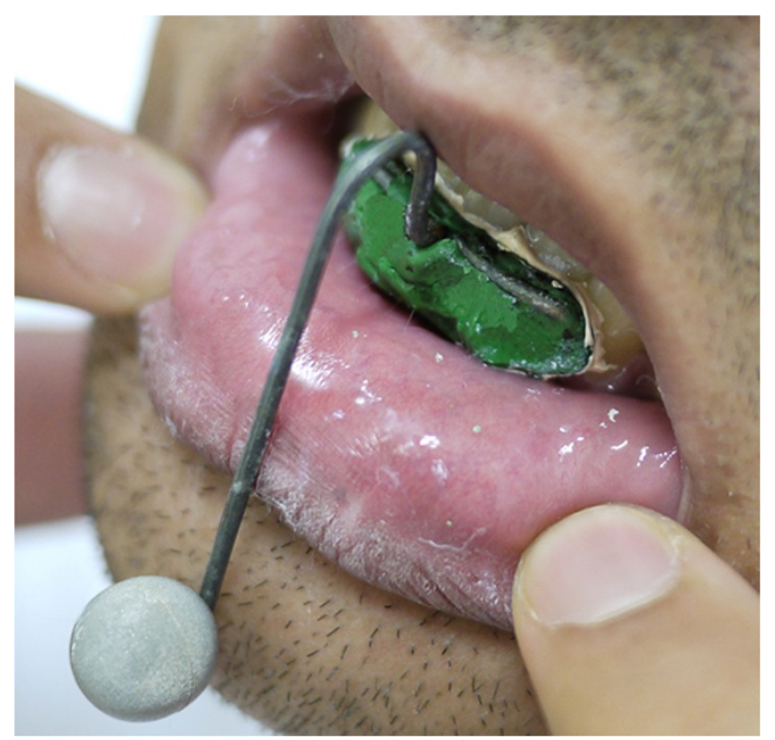

Figure 2 Primary marker. Primary tracking marker is rigidly fixed to the mandible using a metal marker support, which is attached between the inferior lip and the incisors.

enables automatic marker identification using the proposed algorithm that is presented over the next sections. Vertical position was defined above the head line in order to avoid merging of detected points, especially for the central camera. Note that the central camera can see all the nine markers, but the left and right cameras cannot see the markers on the opposite side. A sample of the detected points is illustrated in Figure 4.

\section{Camera calibration}

Before calculating three-dimensional data, cameras need to be calibrated. Camera calibration is a widely used procedure in computer vision for extraction of metric information

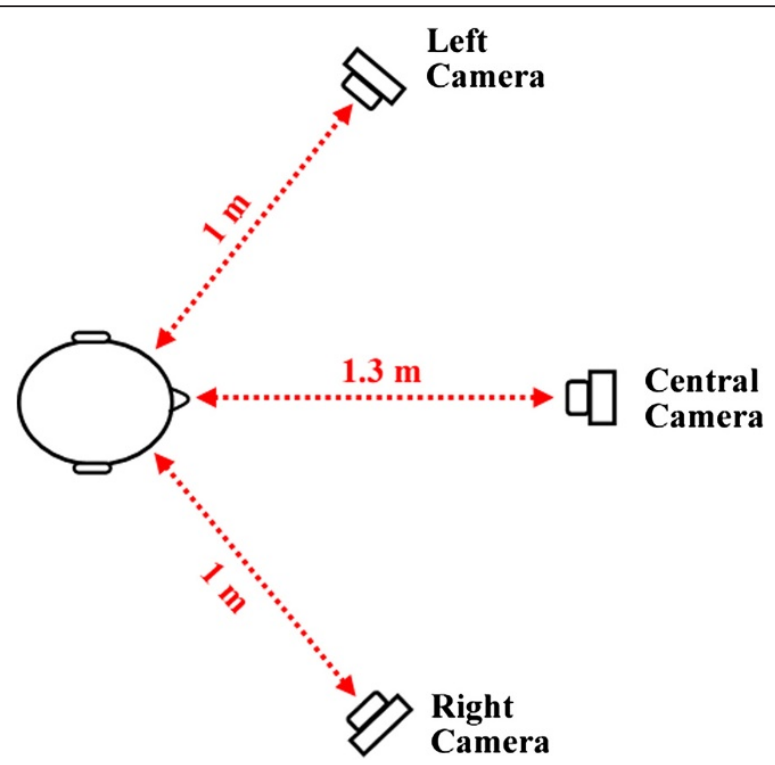

Figure 3 Top view of the proposed camera setup. The left and right cameras are placed at 1 meter distance from the subject forming an angle of 120 degrees. The central camera is positioned at 1.3 meters distance. 


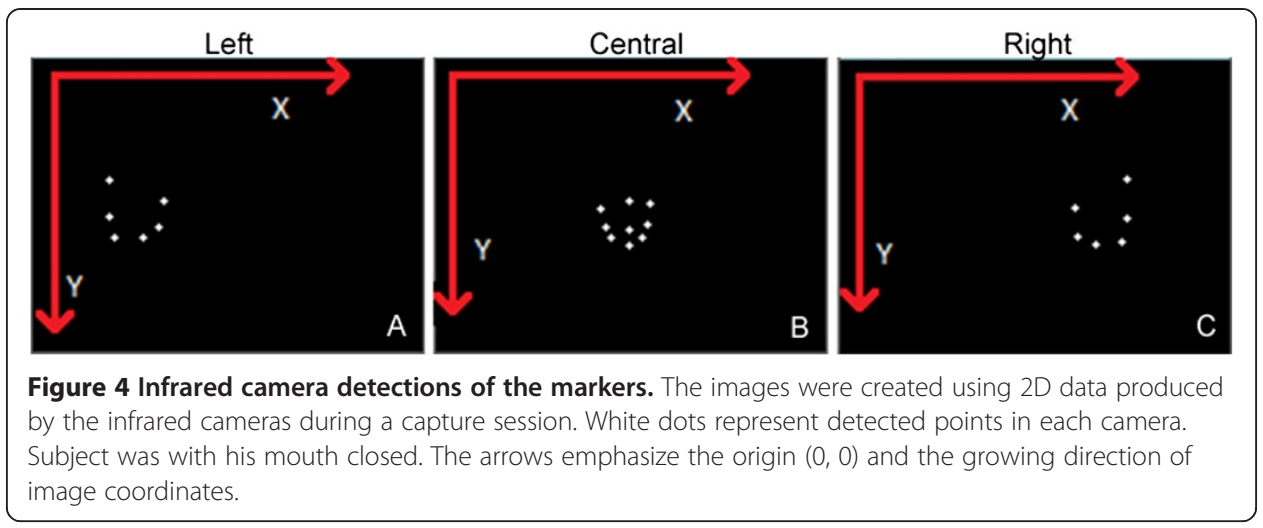

from the scene where the images will be taken. The process aims to find out the exact position and orientation of the camera in space, as well as the camera internal aspects, like focal length and image sensor position. All these parameters allow one to define a mathematical correspondence between the coordinates from the image plane (given in pixels) and the world space coordinates (given in some unit of length). From the several calibration techniques currently available [26,27], we decided to implement the well-known Direct Linear Transformation (DLT) approach [28], since it is relatively simple and provides good precision for 3D calculation when working with small reconstruction volumes [29].

DLT calibration requires the 3D coordinates of at least six points in the scene. To provide this, calibration tools keeping markers at well-known positions are commonly used. For our system, we designed a calibration tool composed by a soldered stainless steel orthogonal triad with seven attached $10 \mathrm{~mm}$ markers, as illustrated in Figure 5. When calibrating the system, the calibration tool must be briefly positioned (using a tripod, for example) in the region where the movements will be taken so that each camera can register at least one image of it. To maximize accuracy and precision, after calibration, mandibular movements must be executed inside the region defined by the $x-y-z$ axes. The subject's head must be oriented so that its Frankfort horizontal plane [30] keeps parallel to the xz plane of the triad

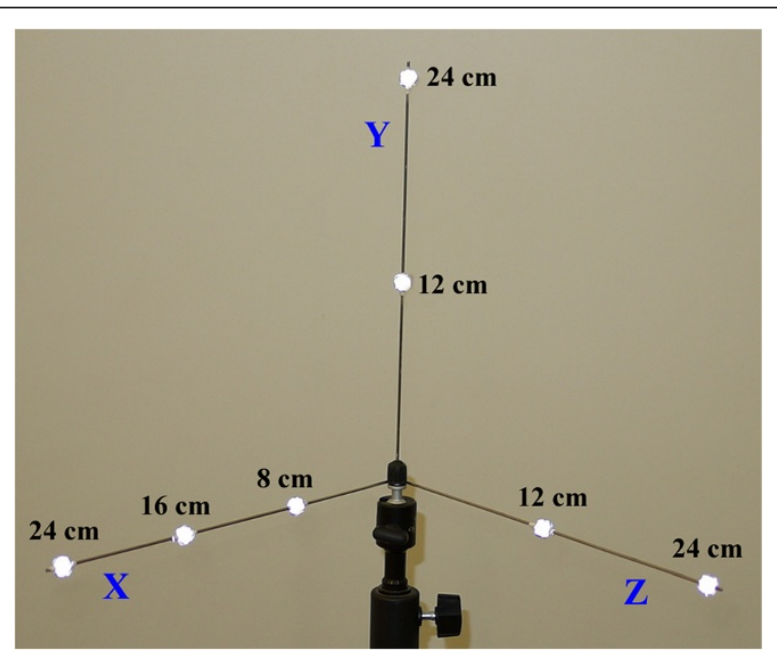

Figure $\mathbf{5}$ Calibration tool designed for the system. Three markers are used in $x$-axis, separated by 8 centimeters from each other, and two markers are used in each remaining y and z-axis. 
and its sagittal plane keeps parallel to the plane defined by the $\mathrm{y}$ and $\mathrm{z}$ axes. Real-time computed 3D coordinates for the secondary markers on TMJ region can be used to support this head alignment by suggesting an eventual correction in its rotation or tilt. Such head positioning should be accomplished by a dental specialist.

\section{Point identification, tracking and 3D reconstruction}

The proposed computational method for automatic recognition of image points and calculation of spatial trajectories of the markers can be organized in three main stages: (1) 2D point identification and inventory, (2) 2D point tracking and (3) 3D reconstruction. These stages are now discussed.

\section{D Point identification and inventory}

Before calculating 3D coordinates of the markers, detected points in different images must first be matched. That means we need to find out which points have been generated by the same reflective marker. Groups of 2D points satisfying this condition become ready to be used in 3D computation. In fact, the properties and constraints of epipolar geometry [31] could be used to solve this point correspondence problem. However, the point identification itself would remain unsolved. Instead, we propose two simple and direct algorithms to solve both correspondence and point identification problems by analyzing the image pattern of each camera (as illustrated in Figure 4, it is expected a specific pattern for each camera). The algorithm for the central camera is presented below:

a. Sort the image points by their $\mathrm{x}$-coordinates in order to organize them from left to right. By analyzing the ordered points, we can identify three groups: (1) three points in the right side of the face; (2) three points in the middle region (forehead, upper jaw and chin) and (3) three points in the left side;

b. Sort the three points with the lowest $\mathrm{x}$-coordinates (group 1) by their $\mathrm{y}$-coordinates and labels the resulting points, respectively, as TMI_RIGHT, ANGLE_RIGHT and MID_JAW_RIGHT;

c. Sort the three points with the highest $\mathrm{x}$-coordinates (group 3) by their $\mathrm{y}$-coordinates. The ordered points must be labeled, respectively, as TMJ_LEFT, ANGLE_LEFT and MID_JAW_LEFT;

d. Sort the three intermediate points (group 2) by their y-coordinates. Labels the ordered points, respectively, as FOREHEAD, UPPER_JAW and CHIN.

The algorithms for the left and right cameras are analogous. Thus, we described just one of them (left camera):

a. Sort the points by their $\mathrm{x}$-coordinates in order to organize them from left to right. By analyzing the ordered points, we can identify two groups: (1) the three left-most points, which match the markers in middle region of the face (forehead, upper jaw and chin) and (2) the remaining three points in the left side of the face.

b. Sort the three points with the lowest $x$-coordinates by their $y$-coordinates. These points correspond, respectively, to FOREHEAD, UPPER_JAW and CHIN;

c. Sort the three points with the highest $x$-coordinates by their $y$-coordinates. The first point in the resulting list must be labeled as TMJ _LEFT; 
d. Sort the last two points without label by their $\mathrm{x}$-coordinates. The first ordered point must be labeled as MID_JAW_LEFT and the second, as ANGLE_LEFT.

Although the point identification algorithms previously described are able to recognize all expected $2 \mathrm{D}$ points, the method itself is a bit time consuming since it involves several sorting operations. Hence, we propose the execution of these algorithms for only the two initial frames of each capture session. For the following frames, however, the point identification can be performed more efficiently by means of $2 \mathrm{D}$ point tracking.

\section{D Point tracking}

For the next camera frames, it is possible to accelerate the point recognition by looking at the neighborhood of each $2 \mathrm{D}$ point identified in previous frames. As the subject is not supposed to do large range movements with the head or condyles, neither big changes in 2D detections nor occlusion of any marker during the capture session are expected. In fact, the tracking technique we suggest is a simplification of that presented by Herda et al. (2001) [32]. It assumes that the displacement of a 2D point from one frame to the next is very small, which allows us to estimate its coordinates in current frame from its coordinates in the past ones.

Considering that $F_{k}^{t}$ stands for a frame captured at instant $t$ from camera $k$, the displacement of a point from $F_{k}^{t-2}$ into $F_{k}^{t-1}$ can be used to get the point's direction and project its expected position $p$ in $F_{k}^{t}$. Once we have this predicted position, we must find the point that is closest to $p$. This point is assumed to be a detection of the same marker and must receive the corresponding label. This is illustrated in Figure 6.

Equations 1 and 2 show the direct calculation of the predicted position $p$. Terms $a$ and $b$ represent, respectively, the coordinates of the tracking point in $F_{k}^{t-2}$ and $F_{k}^{t-1}$.

$$
\begin{aligned}
& p_{x}=b_{x}+\left(b_{x}-a_{x}\right) \\
& p_{y}=b_{y}+\left(b_{y}-a_{y}\right)
\end{aligned}
$$

\section{D Marker reconstruction}

Three-dimensional reconstruction is computed based on the DLT method. As soon as 2D points become identified, those with the same identification (same label) must be used to triangulate the 3D coordinates of the corresponding physical markers. As the markers on the left side of the face (TM), angle and mid jaw) are seen by only the left and central cameras, the pair of $2 \mathrm{D}$ points for each of these markers is employed for

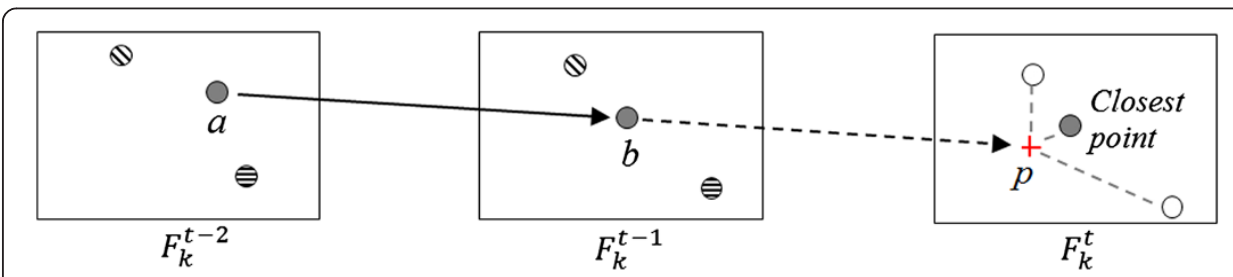

Figure 6 Illustration of 2D point tracking algorithm. The gray point in $F_{k}^{t-1}$ represents the marker being tracked. The predicted position $p$ is calculated from the last positions $a$ and $b$. The technique is a simplification of that presented by Herda et al. [32], since no marker occlusion or merge is expected. 
composing a DLT-based overdetermined linear system whose solution will lead to the related spatial coordinates.

This system can be expressed as the matrix multiplication of the Equation 3. $L_{i}^{l}$ and $L_{i}^{c}$ stand for the i-th DLT coefficient of the left and central cameras, respectively, and $u^{l}$ and $v^{l}$ (resp. $u^{c} v^{c}$ ) are the coordinates of a point in the image captured by the left camera (resp. central). An exact solution for this system is not possible, but we can find a solution that minimizes the residual error by using the SVD technique [33].

$$
\left[\begin{array}{ccc}
\left(L_{1}^{l}-u^{l} L_{9}^{l}\right) & \left(L_{2}^{l}-u^{l} L_{10}^{l}\right) & \left(L_{3}^{l}-u^{l} L_{11}^{l}\right) \\
\left(L_{5}^{l}-v^{l} L_{9}^{l}\right) & \left(L_{6}^{l}-v^{l} L_{10}^{l}\right) & \left(L_{7}^{l}-v^{l} L_{11}^{l}\right) \\
\left(L_{1}^{c}-u^{c} L_{9}^{c}\right) & \left(L_{2}^{c}-u^{c} L_{10}^{c}\right) & \left(L_{3}^{c}-u^{c} L_{11}^{c}\right) \\
\left(L_{5}^{c}-v^{c} L_{9}^{c}\right) & \left(L_{6}^{c}-v^{c} L_{10}^{c}\right) & \left(L_{7}^{c}-v^{c} L_{11}^{c}\right)
\end{array}\right] \quad\left[\begin{array}{c}
x \\
y \\
z
\end{array}\right]=\left[\begin{array}{c}
u^{l}-L_{4}^{l} \\
v^{l}-L_{8}^{l} \\
u^{c}-L_{4}^{c} \\
v^{c}-L_{8}^{c}
\end{array}\right]
$$

For the right markers, the same process can be applied and for the central markers (forehead, upper jaw and chin), 3D reconstruction is achieved using the three detections of each marker to mount a similar linear system with six linear equations. Note that point identification, tracking and $3 \mathrm{D}$ reconstruction process is able to return the spatial coordinates of the nine considered markers for each instant $t$. So, the desired 3D trajectories through time can be generated directly from these reconstructed points. A detailed description about using DLT to compute 3D coordinates can be found in [34].

It is important to mention that during a motion capture session, the subject must keep the head as immobile as possible. In our experiments, this requirement was easily met by using a high-density foam support touching the occipital region of head and neck for head partial immobilization. All participants of these experiments provided written informed consent to participate and the study was approved by the Ethical Committee of Federal University of Uberlandia, Brazil (process number 010/10).

\section{D Data Pre-processing}

3D trajectory of the primary marker can be smoothed using a digital Butterworth filter with 4 poles and a cut-off frequency of $8 \mathrm{~Hz}$. According to Miles (2007) [35], the mandible voluntary movements together with tremors can reach a frequency of 6-7 Hz. Therefore, noise components with frequency greater than $8 \mathrm{~Hz}$ are attenuated, giving a smoother appearance to the movement trace.

\section{Software implementation aspects}

A prototype software was implemented to communicate with the cameras and perform all the tasks previously described, including camera calibration, 2D point match, marker identification, tracking and $3 \mathrm{D}$ reconstruction. The software was programmed using the Microsoft .NET Framework with C\# language and all 3D objects and trajectories were rendered in real-time using the Microsoft DirectX SDK. We have used Intel's OpenCV library to solve the overdetermined linear systems and the experiments were performed in a desktop computer powered by a quad-core $2.8 \mathrm{GHz}$ Intel processor with 4 GB of RAM. 


\section{Results}

\section{System validation and reliability}

The validity and reliability of motion analysis systems have been evaluated in terms of precision, accuracy and repeatability [36,37]. In order to estimate the accuracy and precision of the proposed system, we performed a classical experiment [6,38-40] by using a reference rigid bar of $70 \mathrm{~mm}$ length with two markers attached to its extremities (we implemented a software procedure to track the $2 \mathrm{D}$ points and reconstruct the corresponding 3D coordinates). The bar was moved randomly through the measurement volume during 30 seconds and the distance between the markers was calculated for each frame. The estimated distance values were then compared to the known (true) value of $70 \mathrm{~mm}$. The accuracy was evaluated by determining the root-mean-square (RMS) error associated to the measured distances and the precision was estimated by computing the standard deviation of those distances, the maximum distance error and the 95\% confidence interval. The calculated values are presented in Table 1 and the employed bar is illustrated in Figure 7.

Test-retest reliability was quantified by performing the rigid bar test three more times at intervals of one week. Right before starting each test session, cameras were set-up according to the distances and positions we have proposed. The calibration procedure was conducted every time and after each session the system was disassembled again. For the computed bar length, analysis of variance (ANOVA) test revealed no significant differences across the tests $(\mathrm{p}>0.24)$. The standard deviation of the measurements was less than $0.2 \mathrm{~mm}$ for all sessions and the distance mean error was always less than $0.16 \mathrm{~mm}$.

\section{Clinical experiments}

To investigate the clinical application of the system, it was used to evaluate mandibular movements in a group of 22 subjects. According to Research Diagnostic Criteria for Temporomandibular Disorders (RDC/TMD) questionaire, 20 of these subjects presented no signs or symptoms of TMD and two subjects evidenced masticatory dysfunction.

When starting the capture, the subjects were instructed to sit down in a long back chair with the head supported by a rigid foam. The goal was to help them sense the head position from proprioceptive stimulus and avoid head displacement while executing mandibular movements. They were oriented to execute three types of jaw movements: (1) maximum opening-closing, (2) maximum lateral excursions (left and right) and (3) maximum protrusion, which are all specific movements for the functional evaluation of the mandible. In order to acquire the average parameters, the subjects were oriented to repeat each type of movement 6 times, resulting in 24 cycles.

Table 1 Results of the system validation test

\begin{tabular}{lllllll}
\hline $\begin{array}{l}\text { True 3D } \\
\text { distance }(\mathbf{m m})\end{array}$ & $\begin{array}{l}\text { Estimated } \\
(\mathbf{m e a n}) 3 \mathrm{D} \\
\text { distance }\end{array}$ & $\begin{array}{l}\text { RMS accuracy } \\
\mathbf{( \mathbf { m m } )}\end{array}$ & $\begin{array}{l}\text { Standard } \\
\text { deviation }(\mathbf{m m})\end{array}$ & $\begin{array}{l}\text { Minimum } \\
\text { distance }(\mathbf{m m})\end{array}$ & $\begin{array}{l}\text { Maximum } \\
\text { distance }(\mathbf{m m})\end{array}$ & $\begin{array}{l}\mathbf{9 5 \%} \mathbf{C l} \\
(\mathbf{m m})\end{array}$ \\
\hline 70.000 & 70.156 & 0.259 & 0.208 & 69.501 & 70.683 & 0.481 \\
\hline
\end{tabular}

A reference rigid bar of $70 \mathrm{~mm}$ length with two markers was moved through the reconstruction volume during 30 seconds and the 3D distance between the markers was estimated for each image frame. 


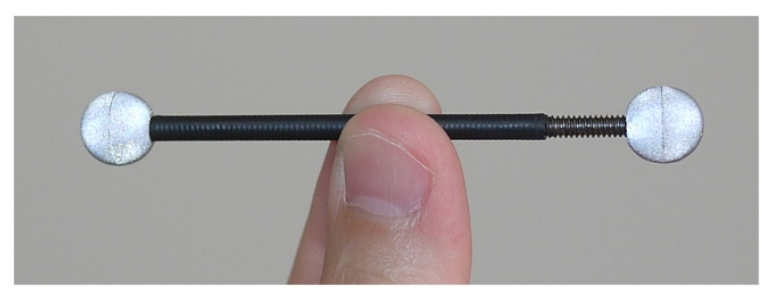

Figure 7 Rigid bar of $70 \mathrm{~mm}$ used to evaluate accuracy and precision.

For all subjects, the system was able to automatically detect the markers, track them and reconstruct their 3D coordinates frame by frame. The corresponding 3D model was always available for real-time visualization and analysis. Figure 8 shows the reconstructed markers and the trajectory travelled by the primary marker during an opening-closing movement performed by a subject without symptoms of temporomandibular dysfunctions. Blue points correspond to spherical markers. Figure 8(A) shows the markers when the mouth was in maximum aperture. The opening trajectory defined by the primary marker is presented in red line and the complete trajectory is presented from different angles and zooms in Figure 8(B) and Figure 8(C).

Figure 9 shows the trajectory of the primary marker along an opening-closing jaw movement executed by a subject with symptoms of TMD. The 3D trajectory is presented from lateral (Figure 9(A)), perspective (Figure 9(B)) and frontal (Figure 9(C)) views. The closing trajectory provides evidence for the abnormal function of the temporomandibular joint, since the lower jaw suddenly goes out of the expected track when finishing the movement cycle.

Table 2 presents the mean values of the maximum displacement registered by the system in each type of jaw movement. Means were calculated for the 20 healthy subjects, where OC-VMax stands for vertical (y-axis) displacement in the maximum aperture of the mandible during opening-closing movement and OC-DMax stands for depth (z-axis) displacement in the maximum aperture of the same movement. Similarly, LL-HMax stands for horizontal (x-axis) displacement in the maximum left lateral excursion. RL and PR stand for right lateral and protrusion, respectively. A dentistry specialist selected the start, the maximum, and the end points for each cycle of movement in the program.

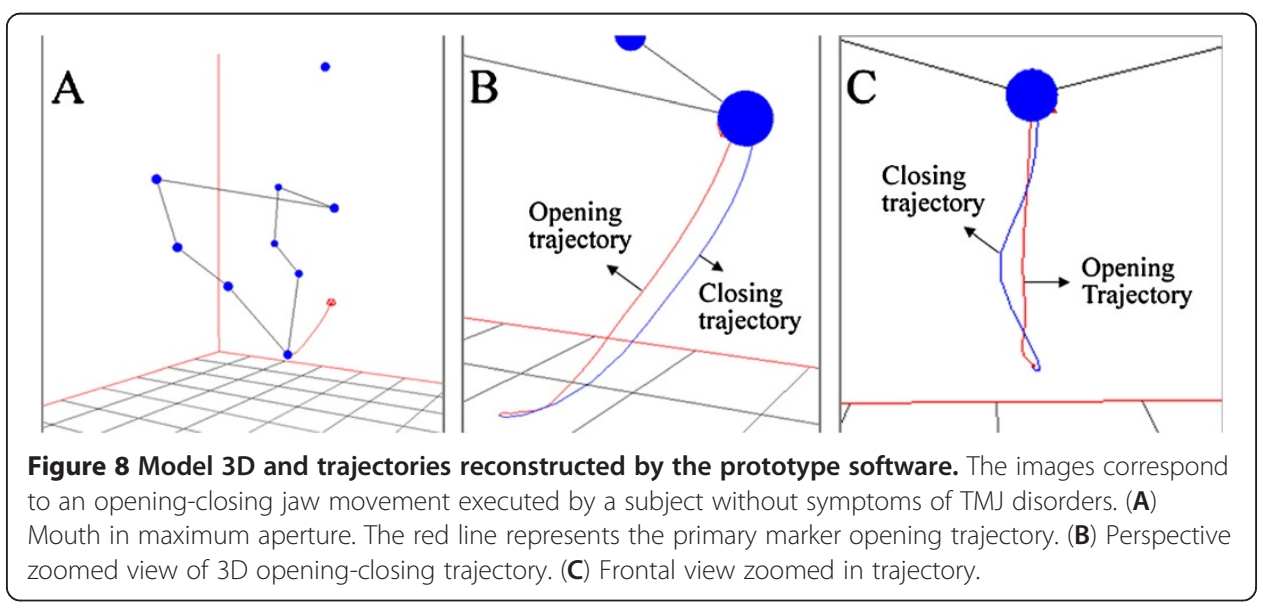




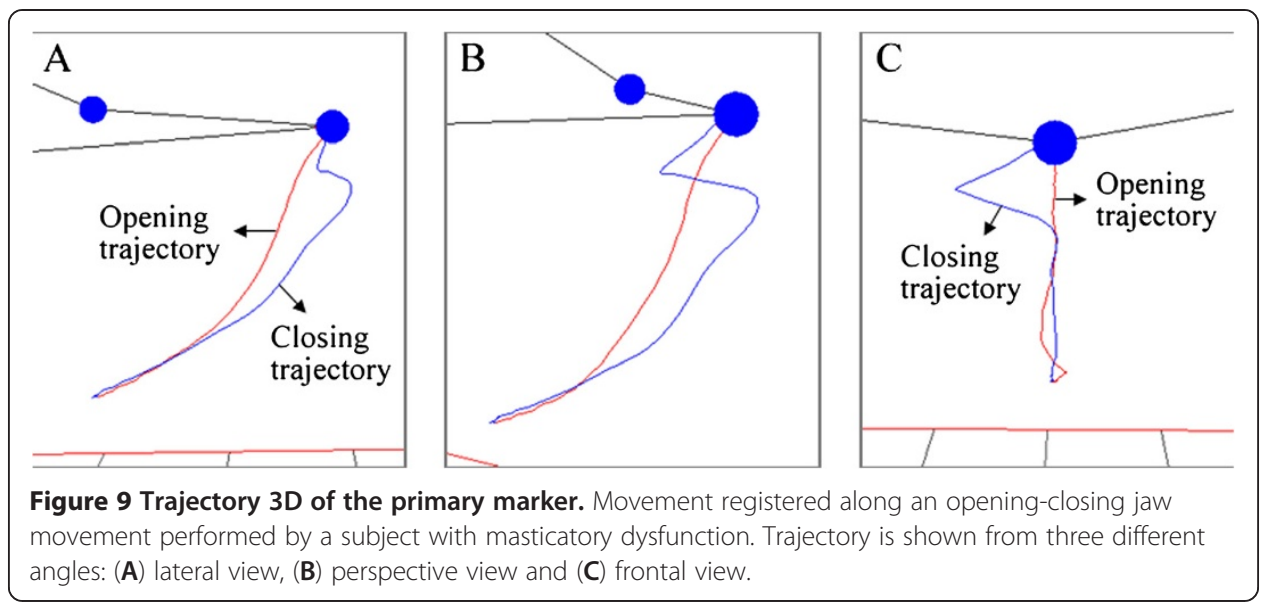

\section{Discussion}

The registered jaw movements for the 20 healthy subjects were considered normal, with trajectories and parameters according to expected patterns and ranges [3,8,22]. For the subject with symptoms of TMD whose data were previously presented, the reconstructed 3D trajectory revealed the severity of the disorder and the exact behavior of the temporomandibular joint when the mandible went out of the expected path. These data can be used by the specialist for a better clinical assessment and for monitoring the evolution of the proposed treatment.

As presented before, the primary marker fixing technique guarantees a precise tracking of the incisal point. Anyway, this is somewhat invasive method, which can cause a minimal discomfort to the subject and consume a bit of time during the fixing process. Depending on the type of movement being captured and the purpose of the experiment, a small displacement error could be tolerated by putting the primary marker easily and directly over the chin, without the metallic support. According to Dworkin and LeResche (1992) [41], for example, lateral displacement of chin and lower

Table 2 The means of the maximum displacement of the primary marker

\begin{tabular}{lc}
\hline Movement & Mean Value (mm) \\
\hline OC-HMax & $1.31 \pm 0.61$ \\
OC-VMax & $39.04 \pm 7.69$ \\
OC-DMax & $27.76 \pm 9.60$ \\
LL-HMax & $9.50 \pm 3.13$ \\
LL-VMax & $3.73 \pm 1.18$ \\
LL-DMax & $1.97 \pm 1.29$ \\
RL-HMax & $9.56 \pm 3.42$ \\
RL-VMax & $3.41 \pm 1.26$ \\
RL-DMax & $1.56 \pm 1.37$ \\
PR-HMax & $1.47 \pm 1.66$ \\
PR-VMax & $3.63 \pm 2.17$ \\
PR-DMax & $7.29 \pm 2.45$ \\
\hline
\end{tabular}

The values were calculated in the axes $x$ (horizontal), $y$ (vertical) and $z$ (depth) for each type of movement for the functional evaluation of the mandible. 
jaw is of similar magnitude, as well as the chewing rhythm, which make the over skin marker a possibility when capturing these parameters. This non-invasive measuring system would enable a patient perform a masticatory movement under almost natural conditions [14].

As presented in Table 1, when validating the system we found an RMS accuracy of $0.259 \mathrm{~mm}$ and a precision of $0.208 \mathrm{~mm}$ within the volume defined by the calibration triad. Although this accuracy value is higher than the $0.198 \mathrm{~mm}$ reported by Fang and Kuo (2008) for their device [19] and greater than those published for the JMA ultrasonic system [20,42], the proposed method has the advantage of being much less obtrusive than these systems. When compared to other electromagnetic and optical techniques $[8,14,43]$, including the previous 2D optical system described by Pinheiro et al. (2011) [6], the proposed system has presented similar or better trueness and precision with the additional capability of supporting real-time assessment. Furthermore, the need to perform the calibration procedure for each new capture session in [6] does not occur in the current proposal. In addition, low maximum error values acquired during the validation test (see Table 1), as well as the $95 \%$ confidence interval for the distance error confirm the reliability and robustness of the proposed system. Table 3 summarizes the properties of some of the previously mentioned techniques and commercial systems in comparison to our method.

\section{Conclusions}

In this paper, we presented a specialized optical motion capture system for analysis of human mandibular movements using three infrared cameras and a set of nine reflective markers. The proposed system can be made with a moderate cost and provides real-time $3 \mathrm{D}$ reconstruction and analysis. Furthermore, it causes a minimum obstruction, can give parameters of facial morphology and can automatically recognize the markers.

To evaluate the algorithms and computational methods, we developed a prototype software and the entire system was employed to reconstruct several movements for the functional evaluation of the mandible in a group of 22 subjects. The system was able to automatically recognize the markers, reconstruct their spatial coordinates and track them in all sessions. The spatial trajectories computed for the incisal point were effectively used to confirm the normal function of TMJ in 20 subjects and to highlight the abnormal behavior of temporomandibular joint in the remaining two. Measured displacements in sagittal, frontal and horizontal planes for each type of movement were in agreement with expected ranges. The measured RMS accuracy of the system was $0.259 \mathrm{~mm}$.

The proposed system is an alternative to the existing optical, mechanical, electromagnetic and ultrasonic-based methods. By using specialized infrared cameras, the system benefits from high accurate marker detection and good capture frequency. At the same time, sophisticated and expensive commercial motion capture software may be dispensed, considering that the proposed computational methods and algorithms are relatively simple and can be implemented with reduced cost. The main goal of the system is to assist specialists in diagnostic and treatment of temporomandibular disorders, since simple visual inspection is subjective and may not be sufficient for a precise assessment of temporomandibular joint and associated muscles. 
Table 3 A comparison between the proposed system and some recently published techniques and commercial systems

\begin{tabular}{|c|c|c|c|c|c|c|c|c|c|}
\hline & 3D analysis & Real-time & Cost & Mean error $(\mathrm{mm})$ & RMS error $(\mathrm{mm})$ & $\begin{array}{l}\text { Overall } \\
\text { precision }(\mathrm{mm})\end{array}$ & $\begin{array}{l}\text { Mandibular } \\
\text { morphology } \\
\text { estimation }\end{array}$ & Obstruction & $\begin{array}{l}\text { Specialized for } \\
\text { mandibular } \\
\text { analyzing }\end{array}$ \\
\hline Proposed System & yes & yes & moderate & 0.156 & 0.259 & 0.208 & yes & low & yes \\
\hline Optical by Pinheiro et al. (2011) [6] & no & no & low & 0.400 & - & 0.300 & no & low & yes \\
\hline Optical by Fang and Kuo (2008) [19] & yes & - & moderate & 0.177 & 0.198 & 0.096 & no & high & yes \\
\hline Optical-CT by Koseki et al. (2007) [14] & yes & no & high & 0.200 & - & - & yes & low & yes \\
\hline Magnetic by Santos et al. (2008) [1] & yes & no & low & - & - & - & - & high & yes \\
\hline Magnetic by Yoon et al. (2006) [8] & yes & no & moderate & 0.320 & - & 0.600 & no & moderate & no \\
\hline Ultrasound JMA [20,42] & yes & yes & moderate & $\sim 0.1$ & - & $<0.1$ & no & high & yes \\
\hline Commercial high-end mocap systems & yes & yes & high & $<0.1$ & $<0.1$ & $<0.1$ & yes & low & no \\
\hline
\end{tabular}




\begin{abstract}
Abbreviations
TMJ: Temporomandibular Joint; TMD: Temporomandibular Disorder; DLT: Direct Linear Transformation; SVD: Singular Value Decomposition; OC: Opening-Closing; LL: Left Lateral excursion; RL: Right Lateral excursion; PR: Protrusion; VMax: Maximum vertical displacement; HMax: Maximum horizontal displacement; DMax: Maximum depth.
\end{abstract}

\title{
Competing interests
}

The authors declare that they have no competing interests.

\section{Authors' contributions}

All authors participated in the research design, data analysis and data interpretation. DAF also participated in algorithms developing, software implementation and manuscript writing. AAP also participated in system design, data collection and manuscript revision. AOA also participated in data collection and manuscript revision. DPBJ also participated in calibration tool construction, data collection and manuscript revision. All authors read and approved the final manuscript.

\section{Acknowledgments}

The authors would like to thank the Brazilian agencies FAPEMIG and CAPES for their financial support for this project (FAPEMIG process number TEC-APQ-01892-11).

\section{Author details}

'Laboratory of Biomedical Engineering, Faculty of Electrical Engineering, Federal University of Uberlandia, Av. Joao Naves de Avila, 2121, Uberlandia, MG CEP 38408-100, Brazil. Department of Occlusion, Fixed Prosthesis and Dental Materials, Faculty of Dentistry, Federal University of Uberlandia, Av. Pará, 1720, Uberlandia, MG CEP 38400-902, Brazil.

Received: 21 October 2012 Accepted: 25 January 2013

Published: 22 February 2013

\section{References}

1. Santos IC, Tavares JM, Mendes JG, Paulo MP: A prototype system for acquisition and analysis of 3D mandibular movement. Int J Mech Mater Des 2008, 4(2):173-180.

2. Naeije M: Local kinematic and anthropometric factors related to the maximum mouth opening in healthy individuals. J Oral Rehabil 2002, 29(6):534-539.

3. Fukui T, Tsuruta M, Murata K, Wakimoto Y, Tokiwa H, Kuwahara Y: Correlation between facial morphology, mouth opening ability, and condylar movement during opening- closing jaw movements in female adults with normal occlusion. European Journal of Orthodontic 2002, 24:327-336.

4. Kalaykova S, Naeije M, Huddleston Slater JJ, Lobbezoo F: Is condylar position a predictor for functional signs of TMJ hypermobility? J Oral Rehabil 2006, 33(5):349-355.

5. Soboleva U, Laurina L, Slaidina A: Jaw tracking devices-historical review of methods development. Part I. Stomatologija 2005, 7(3):67-71.

6. Pinheiro AP, Pereira $A A$, Andrade $A O$, Bellomo D: Measurement of jaw motion: the proposal of a simple and accurate method. J Med Eng Technol 2011, 35(3-4):125-133.

7. Pileicikiene $G$, Varpiotas $E$, Surna $R$, Surna A: A three-dimensional model of the human masticatory system, including the mandible, the dentition and the temporomandibular joints. Stomatologija 2007, 9(1):27-32.

8. Yoon H-J, Zhao KD, Rebellato J, An K-N, Keller EE: Kinematic study of the mandible using an electromagnetic tracking device and custom dental appliance: Introducing a new technique. J Biomech 2006, 39(12):2325-2330

9. Soboleva U, Laurina L, Slaidina A: Jaw tracking devices-historical review of methods development. Part II. Stomatologija 2005, 7(3):72-76.

10. Flavel SC, Nordstrom MA, Miles TS: A simple and inexpensive system for monitoring jaw movements in ambulatory humans. J Biomech 2002, 35(5):573-577.

11. Zafar H, Eriksson PO, Nordh E, Häggman-Henrikson B: Wireless optoelectronic recordings of mandibular and associated head-neck movements in man: a methodological study. J Oral Rehabil 2000, 27(3):227-238.

12. Hamlet S, Faull J, Klein B, Aref A, Fontanesi J, Stachler R, Shamsa F, Jones L, Simpson M: Mastication and swallowing in patients with postirradiation xerostomia. Int I Radiat Oncol Biol Phys 1997, 37(4):789-796.

13. Dijkstra PU, de Bont LG, Stegenga B, Boering G: Temporomandibular joint mobility assessment: a comparison between four methods. J Oral Rehabil 1995, 22(6):439-444.

14. Koseki M, Niitsuma A, Inou N, Maki K: Three-dimensional Display System of Individual Mandibular Movement In Complex Medical Engineering. Edited by Wu J, Ito K, Tobimatsu S, Nishida T, Fukuyama H. Japan: Springer; 2007:117-127.

15. Otake Y, Suzuki N, Hattori A, Shigeta Y, Ogawa T, Fukushima S, Kobayashi K, Uchiyama A: Real-time mandibular movement analysis system using four-dimensional cranial bone model. Systems and Computers in Japan 2006 37(8):1-12.

16. Lin Z, Zecca M, Sessa S, Ishii H, Takanishi A: Development of an ultra-miniaturized inertial measurement unit for jaw movement analysis during free chewing. J Comput Sci 2010, 6(8):896-903.

17. Rohrle O, Waddell JN, Foster KD, Saini H, Pullan AJ: Using a motion-capture system to record dynamic articulation for application in CAD/CAM software. J Prosthodont 2009, 18(8):703-710

18. Santos IC, Tavares JM, Mendes JG, Paulo MP: Acquisition and analysis of 3D mandibular movement using a device based on electromagnetic sensors and a neural network. J Med Eng Technol 2009, 33(6):437-441.

19. Fang J-J, Kuo T-H: Modelling of mandibular movement. Comput Biol Med 2008, 38(11-12):1152-1162.

20. Enciso R, Memon A, Fidaleo DA, Neumann U, Mah J: The virtual craniofacial patient: 3D jaw modeling and animation. Stud Health Technol Inform 2003, 94:65-71. 
21. Hassan EA, Jenkyn TR, Dunning CE: Direct comparison of kinematic data collected using an electromagnetic tracking system versus a digital optical system. J Biomech 2007, 40(4):930-935.

22. Buschang PH, Throckmorton GS, Travers KH, Hayasaki H: Incisor and mandibular condylar movements of young adult females during maximum protrusion and lateratrusion of the jaw. Arch Oral Biol 2001, 46(1):39-48.

23. Mani M, Roberts M, Curtis E, Goodwin N, Zatman T, Wilkes AR, Holt C: Motion analysis - a new technology in airway assessment. Anaesthesia 2010, 65(1):98-99.

24. Kim D-S, Choi S-C, Lee S-S, Heo M-S, Huh K-H, Hwang S-J, Yi W-J: Correlation between 3-dimensional facial morphology and mandibular movement during maximum mouth opening and closing. Oral Surgery, Oral Medicine, Oral Pathology, Oral Radiology, and Endodontology 2010, 110(5):648-656.

25. Naeije M: Measurement of condylar motion: a plea for the use of the condylar kinematic centre. J Oral Rehabil 2003, 30(3):6.

26. Seitz SM, Curless B, Diebel J, Scharstein D, Szeliski R: A Comparison and Evaluation of Multi-View Stereo Reconstruction Algorithms. In Proceedings of the: IEEE Computer Society Conference on Computer Vision and Pattern Recognition - Volume 1. IEEE Computer Society 2006, 2006:519-528.

27. Zollner H, Sablatnig R: Comparision of Methods for Geometric Camera Calibration using Planar Calibration Targets. In Digital Imaging in Media and Education, Proc of the 28th Workshop of the Austrian Association for Pattern Recognition. Edited by Burger W, Scharinger J. Hagenberg, Austria: Schriftenreihe der OCG; 2004:237-244

28. Abdel-Aziz YI, Karara HM: Direct linear transformation from comparator coordinates into object space coordinates in close-range photogrammetry, Proceedings of the Symposium on Close-Range photogrammetry; Falls Church: American Society of Photogrammetry; 1971:18.

29. Remondino F, Fraser C: Digital camera calibration methods: considerations and comparisons. In Proceedings of the ISPRS Commission V Symposium: Image Engineering Vision Metrology; Dresden: Institute of Photogrammetry and Remote Sensing; 2006:266-272.

30. Lundstrom A, Lundstrom F: The Frankfort horizontal as a basis for cephalometric analysis. Am J Orthod Dentofacial Orthop 1995, 107(5):537-540

31. Hartley R, Zisserman A: Multiple View Geometry in Computer Vision. New York, NY, USA: Cambridge University Press; 2004.

32. Herda $L$, Fua $P$, Plänkers $R$, Boulic $R$, Thalmann D: Using skeleton-based tracking to increase the reliability of optical motion capture. Hum Movement Sci 2001, 20(3):313-341.

33. Golub G, Reinsch C: Singular value decomposition and least squares solutions. Numer Math 1970, 14(5):403-420.

34. Shapiro R: Direct linear transformation method for three-dimensional cinematography. Res Quart 1978, 49(2):197-205.

35. Miles T: Postural control of the human mandible. Arch Oral Biol 2007, 52(4):6.

36. Liu H, Holt C, Evans S: Accuracy and repeatability of an optical motion analysis system for measuring small deformations of biological tissues. J Biomech 2007, 40(1):210-214.

37. Allard P, Stokes IAF, Blanchi J-P: Three-dimensional analysis of human movement. Champaign, IL: Human Kinetics; 1995.

38. Ehara Y, Fujimoto H, Miyazaki S, Mochimaru M, Tanaka S, Yamamoto S: Comparison of the performance of 3D camera systems II. Gait Posture 1997, 5(3):251-255.

39. Wiles AD, Thompson DG, Frantz DD: Accuracy assessment and interpretation for optical tracking systems. In Medical Imaging 2004: Visualization, Image-Guided Procedures, and Display; San Diego, California, USA. Edited by Galloway RL. Bellingham, Wash., USA: SPIE; 2004:421-432.

40. Frantz DD, Kirsch SR, Wiles AD: Specifying 3D Tracking System Accuracy - One Manufacturer's View. In Bildverarbeitung für die Medizin. Volume 116. Edited by Tolxdorff T, Braun J, Handels H, Horsch A, Meinzer H-P. Berlin: CEUR-WS.org; 2004:234-238.

41. Dworkin SF, LeResche L: Research diagnostic criteria for temporomandibular disorders: review, criteria, examinations and specifications, critique. J Craniomandib Disord 1992, 6(4):301-355.

42. Hugger A, Bölöni E, Berntien U, Stüttgen U: Accuracy of an ultrasonic measurement system for jaw movement recording. France: 35th Annual Meeting of the Continental European Division of the International Association for Dental Research. Montpellier; 1999.

43. Schuler NB, Bey MJ, Shearn JT, Butler DL: Evaluation of an electromagnetic position tracking device for measuring in vivo, dynamic joint kinematics. J Biomech 2005, 38(10):2113-2117.

doi:10.1186/1475-925X-12-17

Cite this article as: Furtado et al:: A specialized motion capture system for real-time analysis of mandibular movements using infrared cameras. BioMedical Engineering OnLine 2013 12:17.

\section{Submit your next manuscript to BioMed Central and take full advantage of:}

- Convenient online submission

- Thorough peer review

- No space constraints or color figure charges

- Immediate publication on acceptance

- Inclusion in PubMed, CAS, Scopus and Google Scholar

- Research which is freely available for redistribution 